\title{
LA FORMACIÓN DEL PROFESOR DE PSICOLOGÍA CLÍNICA. ¿QUÉ TIENE QUE SABER UN PSICÓLOGO CLÍNICO?
}

\author{
Aquilino Polaino Lorente \\ Catedrático de Psicopatología \\ Director del Departamento de Psicología de la Universidad San Pablo-CEU
}

\section{RESUMEN}

El panorama de actuaciones de la política cientifica en las últimas décadas no ofrece un balance halagüeño. Por lo que se refiere a la psicología clínica se denuncia la paradoja de tener que enseñar psicologia clínica sin práctica reconocida y cualificada en las universidades, con la necesaria formación científica y humanista de los profesores en clínica y la previa selección de los alumnos en función de capacidades y status mental. El nivel de postgrado aparece como el objetivo nunca alcanzado por razones muy diffciles de entender. El trabajo termina con la ampliación de la psicología clínica convencional con la denominada psicologla clínica de la salud, más amplia y que presenta exigencia de formación distintas a las actuales.

Paiabras clave: FORMACIÓN DEL PROFESOR DE PSICOLOGIA CLINICA, FORMACIÓN UNIVERSITARIA DE PSICOLLOGOS CLINICOS, LA PSICOLOGIÁA CLINICA DE LA SALUD- 


\section{SUMMARY}

The political decisions about science and university in Spain along last decades have produced a problematic and unsatisfactory status and the next future does not seem optimistic. In respect to clinical psychology is defended that psychology professors can teach and put into practice their knowledge and research in clinics depending of the departments of clinical psychology. The professors must be formed in scientific and humanistic contents; the students must be selected previously to the specialization in clinical psychology (aptitudes and mental status). The post-graduate level in clinical psychology is the always promised and never achieved. News topics in clinical psychology must be represented in the curriculum: those pertaining to clinical health psychology (chronic disease, families and hospitalization).

Key words: TRAINING OF PROFESSOR IN CLINICAL PSYCHOLOGY, UNIVERSITY CURRICULUM IN CLINICAL PSYCHOLOGY, CLINICAL HEALTH PSYCHOLOGY.

\section{LA PSICOLOGÍA CLÍNICA, EN LA ENCRUCIJADA}

Hay cuestiones que, tal vez por la frecuencia de su presentación, acaban por tornarse connaturales y hasta domésticas para las personas cercanas a ellas. A pesar de constituir auténticas paradojas, una vez que se hacen costumbre, suelen ser toleradas y aceptadas socialmente. Con el pasar del tiempo, los ciudadanos se adaptan a ellas, como si natural o forzosamente tuviera que ser así o no pudiera ser de otra forma. $Y$, aunque en modo alguno lo son, su familiaridad y cercania las ha transformado en una especie de acostumbramiento y rutina para quienes conviven con ellas. Esto es lo que suele acontecer con cuestiones que, por su relevancia y significación exigen una atenta reflexión sobre ellas.

El ámbito de la Psicología Clínica -tanto en lo que respecta a la formación de nuevos profesionales como de los profesores que imparten su enseñanza-, constituye, a mi entender, un ejemplo paradigmático de esta encrucijada. 
Es un hecho cierto la pujanza y excelente valoración que de la Psicología Clínica hacen muchos de los alumnos de Psicología, quienes, por otra parte, desean optar, en forma mayoritaria, por esta salida profesional. De hecho, mucho de ellos eligen esta opción, como se ha puesto de manifiesto de forma persistente en los trabajos de diversos profesores universitarios españoles en revistas nacionales de la especialidad.

Pero, ¿disponen del prestigio y reconocimiento sociales de que son deudores?, ¿en qué cuantía se demandan sus prestaciones?, ¿cuántos de ellos logran dedicarse, de forma estable y continuada, al ejercicio de esta profesión, y sobreviven en el intento?, ¿está la sociedad justa y suficientemente informada respecto de qué es 10 que puede y debe atender un psicólogo clínico?, ¿hay en nuestro actual medio social la necesaria "cultura psicológica" como para atender y acoger a estos profesionales?, ¿están satisfechos los psicólogos clínicos con la formación que reciben?, ¿se ha reflexionado en modo suficiente, por parte del profesorado, acerca de cómo formarles mejor?

Las respuestas a muchas de las cuestiones anteriores no son, por el momento, todo lo satisfactorias que deberían ser. En estas circunstancias, es fácil -tal vez demasiado fácil- apelar a argumentos tópicos y sesgados, a prejuicios y estereotipias culturales, que también convendría desvelar mediante las oportunas investigaciones.

Me refiero, por ejemplo, a las atribuciones que sostienen -sin probado fundamento- que los psicólogos clínicos son supuestos "médicos frustrados" que, cuando alumnos, no fueron capaces de ingresar en una Facultad de Medicina o que la mayoría de los que optan por esta salida profesional están motivados por resolver sus propios conflictos y/o trastornos comportamentales y que, por tanto, su aparente interés es un interés fingido, un interés mediatizado y guiado por sólo su anhelo de resolver sus propios problemas (autoterapia), y/o conocerse mejor a sí mismos.

El imaginario colectivo nacional es muy rico en estas $y$ otras parecidas atribuciones, pero eso sí, sin haber verificado o falsado ninguna de tales hipótesis y, por consiguiente, sin que estén dotadas de validez explicativa alguna. De aquí que, en esta colaboración, 
convenga formular otras posibles cuestiones en una indagación inicial de estos y otros hechos que son pertinentes para lo que aquí interesa.

¿A qué se debe esta mayoritaria opción del alumnado por la Psicología Clínica?, ¿es acaso una cuestión suscitada por la mera curiosidad, por el profundo misterio de los trastornos del comportamiento y las enfermedades o tal vez porque se ha puesto de moda la profesión de psicólogo clínico?, ¿no se tratará quizás de la búsqueda, por una importante porción del alumnado, de un ámbito en el que realizar su ejercicio profesional, con total independencia y responsabilidad, y sin necesidad de dar cuentas a nadie?, ¿no estará influyendo en todo esto el "culto al Yo" y a una presunción idealizada de las relevantes consecuencias que comporta este ejercicio profesional?, ¿cuál es el peso realista de las expectativas económicas concebidas respecto del desenvolvimiento en la práctica de esta profesión?, ¿no será que algunos de ellos han magnificado el quehacer propio de la Psicología Clínica, cuyas exigencias, dificultades y responsabilidades, por otra parte, ignoran?

Es posible que algunas de las anteriores cuestiones incidan en el fundamento de esta toma de decisiones y hasta puedan explicarlas en algunos casos. Pero es probable también que tal opción hunda sus raíces en otros fundamentos de muy diversa naturaleza. Este es el caso, por ejemplo, de quienes sencillamente han experimentado en su intimidad la urgida necesidad vocacional de ayudar a los demás a aliviar sus sufrimientos.

En este último caso estaríamos ante la presencia de una mera cuestión vocacional y natural, que nada o muy poco tiene que ver con los supuestos anteriores. El autor de estas líneas no trata de apoyar en esta colaboración su peculiar punto de vista $-y$ mucho menos de imponerlo. Pero hay algunas paradojas que a este respecto aconsejan, cuando menos, una reflexión pormenorizada.

¿Cómo se explica la persistencia y continuidad en el tiempo de la elección de esta opción por el alumnado de Psicología, al mismo tiempo que otras investigaciones ponen de manifiesto -en el seguimiento profesional de los ya licenciados- el escaso número de psicólogos que se dedican por entero al ejercicio profesional de la Psicología Clínica?, ¿cómo explicar, entonces, esta persistente to- 
zudez motivadora del alumnado y la alta probabilidad de frustración de sus posteriores resultados?, ¿es que estamos acaso ante un ejemplo emblemático de "indefensión aprendida"?

¿No será quizá que la formación que recibe el psicólogo clínico resulta insuficiente e insatisfactoria para resolver los problemas con los que luego se encuentra como profesional?, ¿no será que la sociedad demanda del psicólogo clínico lo que éste no está en condiciones de dar?, ¿no será que el propio "mercado" desempeña aqui una importante función autorreguladora del desempeño de estos profesionales, en función de cuáles sean sus aciertos y desaciertos?, ¿no será que es todavía muy escasa la sensibilización social, respecto de la recepción de estos profesionales, a través de lo que el público demanda de ellos?, ¿están satisfechos los usuarios de los servicios que demandan al psicólogo clínico?, ¿es que hay tal vez unidad de criterio en lo relativo a los objetivos, procedimientos, entrenamientos prácticos, estrategias de intervención, calidad y cantidad de los entrenamientos, etc., a cuyo través se forman los psicólogos clínicos en las diversas Facultades de Psicologia?

De comprobarse la relevancia de estas últimas hipótesis, habria que concluir que algo ha de cambiar en la formación de estos profesionales, si de verdad se pretende que la Psicología Clínica preste el servicio que la sociedad, a través de sus usuarios, precisa de ella. En este caso, habría que impulsar un nuevo "vuelco" de los contenidos de las cuestiones apuntadas. No se trata tanto de observar y analizar el comportamiento de los alumnos de Psicología Clínica como de hacer lo propio respecto del plan de estudios (el "sistema") y los Profesores que imparten las enseñanzas de estas disciplinas.

La situación actual invita a ello. En realidad, estamos en una encrucijada cuya vigencia se anuncia para uno o dos lustros, si nos atenemos a ciertas variables como la incorporación al espacio universitario europeo, el modelo de Bolonia o la integración de los psicólogos clínicos entre los profesionales sanitarios. Mirar hacia otro lado o no encarar los problemas frontalmente, con el rigor preciso, constituiria un grave perjuicio para la ciencia de la Psicología Clínica y para el futuro de los actuales psicólogos y de las próximas generaciones. En esta cuestión nos va mucho a todos: nada más y 
nada menos que la salud psíquica, es decir, algo que afecta a la entera sociedad; $y$ que, por eso mismo, todos han de ganar sin que nadie pierda.

\section{LA FORMACIÓN DEL PROFESORADO DE PSICOLOGÍA CLÍNICA}

La formación del profesorado universitario ha estado estrechamente ligada a los planes de estudios que otros han diseñado -casi siempre, por personas del escenario político, con muy poca o ninguna experiencia personal en la vida académica.

De una parte, los criterios para el acceso y selección del profesorado universitario han sufrido en nuestro país, en las últimas décadas, excesivas reformas, contrarreformas y reformas de las reformas, sin que por ello pueda concluirse nada acerca de la optimización de los recursos humanos de que se disponian en el escenario universitario.

Algunos viejos profesores -especialmente, los pertenecientes al Cuerpo de Catedráticos de Universidad- han experimentado y vivido una injusta usurpación de las funciones que desempeñaban, además del vacío al que de facto se condenaba la plaza que ocupaban. Su función en la selección del profesorado ha sido limitada o contrariada por ciertos requisitos, posteriormente promulgados por instancias de menor rango; la relevancia de sus juicios como expertos en determinadas materias, en lo concerniente a programas, planes de investigación, proyectos educativos, etc., se ha visto alcanzada y fracturada por nuevas disposiciones domésticas, que son ahora de obligado y estricto cumplimiento; su pericia ha sido rebajada e igualada a la baja, lo que se opone frontalmente a los criterios por los que se rigió el concurso-oposición al que se presentaron y ganaron. $Y$, lo que es peor, algunos tienen una fina y sutil intuición de que con los próximos cambios que se avecinan -europeos y no europeos- es bastante probable que el funcionariado universitario -los Cuerpos de Catedráticos y Profesores Titulares- se encamine hacia su extinción en el futuro. Esta situación no parece que vaya a mejorar en la actual y caliente perspectiva de la adaptación forzada al "Proyecto Bolonia". 
De otra, la misma estructura departamental ha puesto de manifiesto la imposibilidad de alcanzar los teóricos objetivos que en un principio la legitimaron. Lejos de ello, ha aumentado su rigidez funcional y el malestar de los profesores que forman parte del Departamento, sin que a causa de ello se haya beneficiado la eficacia investigadora del profesorado. Se diría que, en algunos casos, ni siquiera la convivencia entre los profesores de un mismo Departamento se ha hecho por ello más fluida y comprometida en la tarea de compartir un proyecto común de investigación.

A ello hay que añadir que se han diversificado tanto las formas de acceso a los cuerpos docentes que la actual Universidad se presenta como un conglomerado disfuncional y heteróclito, en el que las divergencias son mucho más amplias y frecuentes que las convergencias.

En un caldo de cultivo así, nada tiene de particular que la carga docente de muchos Profesores haya sido diseñada de forma circunstanciada, para salir del paso, ad tempus, de "quita y pon", de apenas un curso escolar -con tal de salvar la dificultosa y urgida situación docente de solucionar el problema docente "este curso"-, para con toda probabilidad comenzar de nuevo el próximo curso académico con otra nueva materia, que jamás ese profesor había explicado, y así sucesivamente. Pero como ya sentenció Varrón, nihil novit, qui aeque omnia, "nada sabe el que todo lo sabe igual" y nemo omnia potest scire, "nadie puede saberlo todo".

El coste de estas deficiencias todavía no se ha calculado. Sería pertinente hacer una auditoria -ahora que tanto se habla de la calidad de enseñanza- acerca del coste social y profesional en los alumnos, como consecuencia de estos cambios vertiginosos en las asignaturas impartidas por-valga el eufemismo- los "profesores por un año".

Mientras tanto, el purismo universitario continúa. A pesar de arreciar las críticas al profesorado de la Universidad -de las que el autor de esta colaboración puede ser una muestra más-, hay que advertir, sin embargo, que lo más granado de la "sustancia pensante" de nuestra sociedad todavía hoy se encuentra en el escalafón de Profesores Numerarios de Universidad. Esta afirmación también es válida respecto de la enseñanza de la Psicología Clínica. Asentado 
este aserto -que es de justicia, contra los que denigran de forma generalizada y sin matización alguna a la totalidad del Cuerpo docente universitario-, es preciso aproximarnos a un cierto diagnóstico de nuestras instituciones universitarias, en lo relativo a la enseñanza de la Psicologia Clínica.

En lo que atañe a la enseñanza de esta compleja materia, hay que afirmar que buena parte del profesorado que la imparte no dispone de la necesaria experiencia práctica que seria menester, experiencia que fundamentalmente se forja en el ámbito asistencial y en el contexto clínico. Es posible que haya algunos Profesores de Psicología Clínica que, encerrados en la torre blindada de su Departamento, se perciban como personas incapacitadas para mostrar a sus alumnos (en el pertinente y concreto ejercicio práctico y de forma satisfactoria), los necesarios resultados verificadores de su supuesta pericia en los conocimientos teóricos que en la actualidad enseñan.

No estoy muy de acuerdo con la corrosiva e impertinente afirmación de algún autor que, a este propósito, Ollero Tassara (1985) cita en su libro- Dice asi: "El que sabe algo, lo hace; si no sabe hacerlo, lo enseña; si tampoco sabe enseñarlo, acaba de bibliotecario; pero si le falta paciencia y es desordenado se hará periodista".

De ordinario, quien sabe algo de clínica, también sabe hacerlo, aunque tal vez debiera optimizar sus destrezas y habilidades prácticas de lo que sabe, en el escenario de la clínica. En cualquier caso, si lo sabe y sabe hacerlo es harto probable que también sepa enseñarlo (aunque también en esto cabe mejorar).

No se trata, pues, de establecer un modelo de profesor non plus ultra, sino simplemente de que el profesor conozca el haz y el envés, la teoria y la práctica de la materia que enseña en Psicología Clínica, de manera que haya una cierta garantía de que su "saber" y su "hacer" son conformes a los contenidos de la disciplina cuya enseñanza imparte. Lo que no es de recibo es que se enseñen unos conocimientos de naturaleza eminentemente práctica desde la pizarra o, en la actualidad, desde el power point $y$, por ahora, nada más.

Por mucha que sea la imaginación de profesores y alumnos, ambos se ven concitados y exigidos por el reto insustituible de la realidad, que es donde en verdad se acuna el quehacer clínico. 
He aquí una de las razones por las que la Psicología, teórica y académica, no puede enseñar lo que no sabe ni jamás ha practicado. Se sostendrá que los Profesores están replegados en una torre de marfil, aislada e incomunicada, desde donde nada resuelven, porque no están en otra realidad que no sea la de la autocontemplación y la autoexaltación.

Tal afirmación tampoco es justa. Muchos de ellos han probado sus conocimientos téricos e investigadores de modo eficiente, aunque tal vez jamás tuvieron la oportunidad de hacer lo propio en el ámbito de lo aplicado.

De otra parte, la misma Universidad está comprometida en esa ausencia de experiencia de algunos de sus Profesores de Psicología Clínica. De hecho, la misma Universidad prohíbe al profesorado lo que por naturaleza debiera exigirle: que revalidara las hipótesis y teorías que explica con su exigente verificación en el contexto clínico de la práctica cotidiana.

Pero en la Universidad se ha optado por la teoría y se ha prohibido la práctica, incluso entre los Profesores que imparten disciplinas de un nítido contenido asistencial como la Psicología Clínica, cuya aplicación práctica es decisiva. En principio, al autor de esta colaboración no le parecería tan mal tal opción, siempre que la teoría enseñada tuviese la oportunidad de verse sometida a las pertinentes refutaciones o verificaciones en el ámbito estrictamente clínico, con independencia de que quienes las expliquen estuvieren comprometidos o no con tal práctica.

Es decir, se trata de volver a observar la naturaleza de aquello que se enseña, sea desde la perspectiva sintomática, fenomenológica, comportamental, neuropsicológica o de cualquier otra de las muchas que se emplean en la clínica. Pues, como afirmaba Sir Francis Bacon (1984): Natura non nisi parendo vincitur, "sólo se ejerce imperio en la naturaleza, obedeciéndola". Es decir, sólo se aprende clínica mediante la observación de lo que naturalmente acontece en los pacientes. La naturaleza de la mayoría de los trastornos y alteraciones no puede ser aprendida sólo en "pacientes simulados", "alumnos voluntarios" o a través de las mil y una imágenes que la poderosa tecnología actual nos proporciona. 
No parece que sea éste el camino a seguir. En la enseñanza de la Psicología Clínica se ha procedido de otro modo: se ha optado por la enseñanza teórica sin posibilidad alguna de enriquecerla con el contraste de la práctica clínica. Cualquier Profesor de este ámbito conoce muy bien la prohibición de simultanear la docencia con el ejercicio profesional, lo que forzosamente $-y$, al menos, potencialle transforma en un "ratón de biblioteca". Se diría que en este contexto se hace lo contrario de lo que aconseja el proverbio azerbaijaní que reza: "habla de lo que entiendas, no sobre lo que hayas leído". Aquí no se habla de lo que se hace o se entiende, sino sólo de lo que se ha leído. $Y$ así nos va.

Por el contrario, lo que solicitan los alumnos de Psicología Clínica -en esto muy atenidos a la realidad- es que la ratio práctica/teoría sea a favor de la primera. Sea por su anhelo de nuevas experiencias, de conocimientos significativos vivenciales y/o experienciales o sea porque desconfían de las teorías - tan cansados como suelen estar de sufrirlas-, el hecho es que exigen un mayor tiempo de entrenamiento en el ámbito de la clínica. Pienso que en este punto su arihelo es tan razonable como difícil de satisfacer, dada la estructura de los actuales $-y$ posiblemente también los futuros- planes de estudio.

Sea como fuere, una persona que no verifica, comprueba o somete a falsación empírica las teorías que enseña, acabará a la postre por ser un mal teórico. Un mal teórico será siempre un mal práctico, porque si la teoria en que se fundamenta la práctica está mal formulada o no ha sido verificada, la práctica derivada de ella con harta probabilidad le cabe la posibilidad de ser errónea. En cambio, un buen profesional práctico tiene más posibilidades de ser un buen teórico, al menos en aquello en que en el contexto de la práctica cotidiana tiene una cierta experiencia y le ha demostrado que genera resultados eficaces.

En este mismo sentido cabe formular algunas cuestiones en lo relativo a la metodología. También al psicólogo clínico se le exige en este punto más de lo que necesita y menos de lo que le es necesario. Así, por ejemplo, del método clínico muchos de ellos ni siquiera han oído hablar. Por el contrario, del método y de los diseños experimentales como del análisis estadístico han tenido que sufrir casi todo. Convendria preguntarse acerca del «para qué» de tal metodología. 
Es cierto, que deben aprender a valorar la validez y fiabilidad de los resultados de que se informa en cualquier artículo y de las hipótesis que contribuyeron a su diseño, de manera que obtengan sus propias conclusiones al respecto $y$, en consecuencia con ellas, apliquen o no aquello que han estudiado. Pero esto no debiera conducirnos en ningún caso a la "divinización de la metodología". También se dan aquí ciertas disonancias. El Profesor de metodología suele ser muy riguroso -escrupuloso casi- en el ejercicio de los análisis y supuestos metodológicos de que parte para sus enseñanzas. Pero en otras muchas ocasiones él mismo no dispone de ningún diseño que haya llevado a la práctica y que metodológicamente sea en su rigor impecable, bien por la elegancia de su diseño o/y por la eficacia de los resultados obtenidos.

Esta paradoja es más demostrativa todavía en lo que se refiere al empleo de los recursos metodológicos que en teoría ha aprendido el psicólogo clínico y que tal vez nunca o muy rara vez use de ellos. Así las cosas, no parece sino que el aprendizaje de las sofisticadas enseñanzas metodológicas sólo sean útiles para quienes han optado por perseguir un puesto docente en la Facultad en que se formaron.

Admitido este supuesto, tal vez convenga responder a otras preguntas inquietantes como las siguientes: De cada promoción de licenciados en Psicología, ¿cuántos de ellos aspiran a ser Profesores universitarios?, ¿cuántos de ellos lo serán algún día?, ¿cuántos de ellos acabarán su doctorado?; por el contrario, ¿cuántos psicólogos clínicos se dedicarán a la investigación en el contexto del ejercicio práctico de la clínica?, ¿cuál es la producción científica de los psicólogos clínicos que no se dedican a las actividades académicas?, ¿por qué se da la paradoja de que el clínico que está en contacto con sus pacientes apenas investiga y, en cambio, sí que investiga el profesor universitario que tan alejado está de los pacientes?, ¿es que acaso todavía no ha llegado la hora de superar esta paradoja, e integrar la investigación y la clínica en el correcto y pertinente marco teórico?

En este nudo gordiano es donde ha de decidirse el futuro de la formación del profesorado de Psicología Clínica. Aunque para llevarlo a cabo sea preciso un cambio radical de las estructuras universitarias. De no iniciarse en la actualidad este giro copernicano 
podría sentenciarse que está definitivamente perdido el futuro de la Psicología Clinica.

La formación del profesorado de Psicología Clínica ha de pasar necesariamente por la práctica clínica. No deja de ser extraño que muchas de las Facultades de Psicología dispongan de laboratorios y animalarios y no estén vinculadas -ni laxa ni estrechamente- $a$ un Centro de Salud. La experimentación animal es, sin duda alguna, necesaria; pero más necesaria es todavía la práctica clínica. De hecho, muy pocos alumnos se dedicarán en su futuro profesional a la experimentación animal, mientras que son mayoría los que parece que se dedicarán al quehacer clínico.

Desde luego que esa formación no se limita a sólo la práctica clínica. El profesorado que tenga a su cargo la responsabilidad de enseñar las disciplinas clínicas -cualesquiera que éstas fuesen- ha de disponer de la suficiente formación antropológica, conocer muy bien las posibilidades de las entrevistas clínicas y las estrategias de evaluación psicológica y psicopatológica, los criterios para el diagnóstico y el diagnóstico diferencial de los diversos procesos, las habilidades de negociación y mediación en las entrevistas y el abordaje de ciertos conflictos, las destrezas en la resolución de problemas y ahondar en todo lo relativo a los factores psicológicos en que ha de intervenir, tanto en lo relativo a las enfermedades orgánicas como psíquicas.

Esto quiere decir que ha de conocer -al menos de un modo general- la mayoría de las escuelas que son relevantes en el ámbito de las psicoterapias, así como sus peculiares indicaciones, la eficacia diferencial de cada uno de esos procedimientos de intervención en los diversos procesos en que se emplean, su coste-beneficio y su coste-eficiencia, etc.

Dada la evolución experimentada por la actual jurisprudencia y lo delicado de ciertas situaciones, sería muy conveniente que los profesores de Psicología Clínica dispusieran de los necesarios conocimientos acerca de las responsabilidades en que, en el futuro, pueden incurrir sus alumnos en el contexto donde llevan a cabo el ejercicio de su profesión. Por último, no debiera omitirse en ellos una sólida formación ética, por cuanto muchas de las decisiones por las que han de optar conviene realizarlas también a la luz y de acuerdo 
con los principios éticos (de justicia, autonomía y benevolencia, principalmente), por los que se rige esta actividad profesional (deontología profesional).

Esta formación genérica ha de constituir un obligado marco de referencias, con independencia del saber y el contenido de la disciplina especializada que se enseñe. En este punto conviene recordar que la práctica clínica constituye un universo demasiado amplio como para que pueda ser abarcado con facilidad por una sola persona. Es preciso, por eso, insistir en la necesidad de la especialización. Pero sin abandonar ese marco de información general en el que cualquier especialidad que se enseñe, por específica que sea, hunde allí sus raíces y se perfecciona gracias precisamente a ese marco de referencias inequívocas.

De la epidemiología a los programas preventivos, de la organización de los equipos de salud al impacto de las diversas enfermedades en la familia, del estrés y la salud laboral a los efectos psicopatológicos de las enfermedades crónicas, una multitud de especialidades se concitan en este universo gígantesco e inagotable que es la Psicología Clínica. Sucede aquí lo que Faraday (17911867) afirmara respecto de la electricidad: "La gran belleza de nuestra ciencia -sostenia- es que su avance, lejos de agotar el campo de investigación, en algún grado abre las puertas a un conocimiento más vasto y diverso, desbordante de hermosura y utilidad".

Resulta un tanto utópico, por eso, que el alumno tratara de abarcar todos estos conocimientos durante la licenciatura (especialmente si se reduce su duración a sólo cuatro años) o que el profesor tratara de exigírselos. Es menester apelar a los estudios del Tercer Ciclo, a los que se hará referencia más adelante.

Baste con lo afirmado líneas atrás para informar al lector de la objetiva complejidad que comporta la formación del profesorado en estas disciplinas, algo que no se puede improvisar y mucho menos alterar de forma significativa -a través de los cambios en los planes de estudios- en apenas un lustro, como ha venido sucediendo hasta ahora.

Conviene recordar que lo que interesa al ciudadano eso es lo que precisamente no suele interesar al Profesor universitario, de la misma forma que lo que a este último interesa es lo que resulta indiferente al ciudadano de a pie. Esto que puede ser hasta justificable en otras 
especialidades de la Psicología -las ciencias básicas, por ejemplo-, resulta mucho más difícil de argumentar en lo relativo a la Psicología Clínica.

En este último ámbito, el diálogo Universidad-Sociedad se presenta mucho más fluido, aunque erizado de dificultades que habrá que superar. Al fin y al cabo de lo que se ocupa la Psicología Clínica es de la salud, y esta es algo que a todos nos interpela y atañe, por cuanto que constituye un bien que en modo alguno es renunciable.

\section{EL RETO UNIVERSITARIO DE UNA DIFÍCIL Y EXIGENTE SÍNTESIS}

¿Si su mujer o su hija precisara de la consulta con un psicólogo clínico, a quien la remitiría Usted, como Profesor universitario de Psicología Clínica? ¿A quién acudiría si barruntara o padeciera Usted un grave trastorno psicopatológico, que le causara mucho malestar? Son éstas preguntas un tanto ingenuas, pero no así la reflexión que han de suscitar y las respuestas que a ellas se den.

Así, por ejemplo, si el citado Profesor no remitiera a su mujer o a su hija a ningún Psicólogo Clínico, tal modo de comportarse constituiría un excelente indicador de la desconfianza que le merecen estos profesionales; profesionales a cuya formación él mismo ha contribuido.

¿Qué cabe esperar de un profesional que desconfía de los profesionales que, cuando alumnos, él mismo formó? Es posible que lo que a este Profesor le suceda es que no cree en la eficacia de la Psicología Clínica, como algunas veces he tenido ocasión de comprobar. Esto, qué duda cabe, es todavía más grave, por cuanto que de mantenerse tal actitud, su misma posición como Profesor se expondría a una situación de grave riesgo, pues estaría deslegitimada. No debieran eludirse estas cuestiones, por elementales que, prima facie, parezcan.

Si los conocimientos teóricos enseñados no pueden ser refrendados en el ámbito de la práctica clínica, entonces es que las teorias en que aquellos se fundamentan no tienen la necesaria consistencia, es decir, que son teorías de dudoso alcance explicativo. Pero, de ser así, ¿en virtud de qué principio continúan enseñándose? 
Si por el contrario, de lo que se duda es de la capacidad de esos profesionales para comportarse en el ámbito de la clínica con el rigor, la pericia y la honestidad que son necesarias, entonces, ¿por qué no se han ajustado a esos objetivos las enseñanzas que se les impartieron?, ¿es que no habría sido menester incluir en el plan de estudios otras materias pertinentes, a fin de que el alumno aprendiera esa pericia, rigor y honestidad que ahora se le exige?

Es posible que su confianza en los psicólogos clínicos aumentara si se hubiera incorporado al curriculum vitae de ese Profesor una cierta experiencia clínica y el hecho de haberla compartido con sus alumnos. De aqui la necesidad irrenunciable de que los Profesores de Psicologia Clínica satisfagan el logro de esa difícil y exigente sintesis curricular entre la docencia y la clínica, una sintesis que exigirá de ellos una mayor y más intensa dedicación.

Esta exigencia viene demandada por el contenido de lo que enseñan, pues conviene no olvidar que la docencia por ellos impartida es al fin una docencia clínica o útil para la clínica, es decir, una docencia en el proceso de enseñanza-aprendizaje, cuyo escenario natural es el contexto clínico.

De hacerse este esfuerzo, de seguro que cambiarian muchas de las penosas circunstancias que hoy sofocan la Psicologia Clínica y su crecimiento como ciencia. Pues como escribe Jay Gould (1984) respecto de la evolución, "ciencia es todo lo que está confirmado de un modo que no sería razonable negarle nuestra aprobación provisional".

En la actualidad, sin embargo, persisten en la Psicologia Clínica quizás demasiadas cuestiones, en las que por esa ausencia de confirmación puede incluso ser razonable que tanto el usuario como los alumnos y profesores les nieguen su aprobación provisional, que es lo propio de la ciencia, según el autor antes citado.

\section{¿QUÉ TIENE QUE SABER UN PSICÓLOGO CLÍNICO?}

Esta es, qué duda cabe, la primera pregunta que hay que formular y a la que hay que responder. Entendemos que el Psicólogo Clínico ha de saber en modo suficiente los contenidos científicos específicos 
de su profesión (formación teórica), pero ha de saber, también de forma obligada, cómo aplicar esos conocimientos (formación práctica) y, al menos, haberse iniciado en las tareas de saber cómo continuar aprendiendo los nuevos conocimientos que se produzcan (formación en investigación), de manera que pueda estar al día en las cuestiones que sean propias del ejercicio de su profesión.

Los tres saberes anteriores no son independientes unos de otros, sino que están entrelazados entre sí y conformados según la densidad y espesor de los innovadores descubrimientos y de los fundamentos antropológicos y éticos cuyo cabal cumplimiento es pertinente en el ejercicio de esta profesión.

Innovación y tradición se aúnan en el quehacer de la clínica. Es preciso incorporar los nuevos descubrimientos, pero sin olvidar jamás la dignidad y el respeto de la persona en que se interviene. En este punto, es de vital importancia reincorporar al sujeto en el contexto psicológico, del que tal vez ha estado exiliado acaso durante demasiado tiempo.

Sin haber participado en ese conocimiento por conriaturalidad que es el propio de la clínica- el psicólogo encontrará serias y graves dificultades no sólo en lo que respecta al ámbito estrictamente científico de su profesión, sino también en su dimensión humana, en el modo de relacionarse con los pacientes, en la forma de preguntar y acoger sus respuestas, en la habilidad con que maneja su propia angustia de exposición, en la prudencia ante ciertas dudosas cuestiones en que no es fácil tomar una decisión.

Algunas de estas y otras características profesionales exigen del psicólogo un gran equilibrio personal, lo que algunos denominan no sé si con acierto o no- una "personalidad madura". Esta relevante cuestión deberia debatirse en un contexto diferente. Dada su amplitud y significación, entiendo que no es éste el lugar apropiado, por lo que renuncio a ello. Pero no me puedo resistir a sugerir una sola cuestión: la que se refiere a los criterios de selección de los alumnos de Psicología Clínica. No estaría de más que se evaluara antes la salud psíquica de los aprendices de Psicología Clínica -mediante, por ejemplo, un elemental screening, de manera que, en la medida de lo posible, se les oriente profesionalmente hacia donde sea más conveniente para ellos y la sociedad, y en evitación de problemas juridicos de lamentables consecuencias. 
Esta cuestión acaso no sea "políticamente correcta" pero, pienso, que es imprescindible para el "desarrollo sostenible" de la actual Psicología Clínica, dada la importancia, cercanía e impacto de la salud psíquica del psicólogo en las cuestiones nucleares de otras personas, con las que ha de habérselas en su futuro profesional.

La restringida limitación temporal de los años de formación en los estudios de la licenciatura en Psicología pone de manifiesto la necesidad de prolongar la formación del psicólogo clínico, de manera que se dé la suficiente aunque mínima cobertura a la diversidad de conocimientos que se integran en esta especialidad.

Es conveniente también que aprendan a reflexionar y a formularse cuestiones que, surgidas a orillas del quehacer clínico y acaso por su elementalidad pudieran ser despreciadas. En la clínica no hay nada despreciable como nada está resuelto de un modo definitivo. Si la clínica es tan inquietante y comporta tanto riesgo es precisamente por esto, porque es una ciencia inabarcable e inacabada, que está siempre abierta a una multitud de mejoras e innovaciones.

He aquí otra razón para que el Profesor esté cerca de sus alumnos y participe con ellos de las experiencias clínicas que viven. Los seminarios de distinta indole, los grupos de trabajo, los talleres especializados y las sesiones clínicas son por este motivo irrenunciables. El Profesor ambicioso aspira a que sus alumnos lo hagan mejor que él, y con ello a que progrese la Psicología Clínica. Este anhelo seria una excelente garantía de la rectitud de su comportamiento magisterial. Tal vez se trata aquí de partir de un concepto más funcionalista y también más generoso del concepto de ciencia. Como escribe Max Gluckman (1965), la "ciencia es toda disciplina en que los tontos de una generación pueden llegar más lejos que los genios de la precedente".

De esto precisamente es de lo que ha de tratar de hacerse de los alumnos de Psicología Clínica, a fin de hacerles avanzar a ellos y a la ciencia que sus Profesores enseñan. Hay sobradas razones para ello. La primera y principal consiste en que detrás de todas esas actividades hay personas que sufren, personas dolientes cuyos sufrimientos y necesidades personales, familiares, grupales o sociales, hay que aliviar. En eso radica también el carácter eminentemen- 
te vocacional y la grave responsabilidad social de quienes se dedican profesionalmente a la orientación, asistencia, ayuda e intervención en "los otros", a cuyo servicio están.

\section{UN FRAGANTE ERROR EN EL DISEÑO CURRICULAR DE SIEMPRE: EL OLVIDO DE LOS ESTUDIOS DE POSTGRADO}

Una cuestión a la que han rehusado entrar todos los planes de estudio, hasta ahora concebidos, es la relativa a las enseñanzas correspondientes al así llamado Tercer Ciclo. Esta apremiante y vigorosa exigencia "se ha dejado siempre para después", pero ese "después" nunca llegó. La disculpa para tal omisión siempre fue rápida y contundente: la escasez de recursos económicos.

Las promesas que se hicieron, a este respecto, han sido una y otra vez incumplidas. No resulta aventurado que algunas profecías acerca del Tercer Ciclo se hayan cumplido. "El 'tercer ciclo' -escribe Ollero Tassara, 1985- puede acabar perdiendo todo contorno definido, para convertirse más bien en un reptante perfil de asistencia a conferencias, trabajitos para Jornadas varias y otras actividades domésticas del Departamento, premiadas todas ellas generosamente con números para el bingo doctoral" (p. 82). Y la profecía, lamentablemente, se ha cumplido en modo suficiente durante las dos últimas décadas.

Más adelante se apeló a los Masters, como modo de paliar el vacío existente, al mismo tiempo que como una estrategia de mero aggiornamento para "modernizar" y financiar nuestras universidades. La iniciativa hizo fortuna y alcanzó un notable éxito empresarial y económico, pero en modo alguno satisfizo las necesidades perentorias de la formación en Psicología Clínica.

El autor de estas líneas no descalifica por ello la natural bondad de algunos de estos Masters. Pero ha de advertir, en primer lugar, que la mayoría de ellos abarcan sólo un restringido y peculiar ámbito de la Psicología Clínica, una casi microespecialidad. Y, en segundo lugar, que algunos de ellos fueron apenas la "flor de un dia" o de un curso, como puede comprobarse si algún estudioso pasara re- 
vista a los programas y folletos anunciantes editados por las diversas universidades.

Ninguna de las opciones anteriores reúnen las condiciones exigidas para la formación clínica-práctica de los psicólogos clínicos. Es decir, las enseñanzas durante el pomposo y tan cacareado Tercer Ciclo están aún por diseñar. ¿Qué habria sucedido si los licenciados hubieran tenido acceso a un Master en Psicología Clínica General, de dos o tres años de duración, en el que un $75 \%$ del tiempo empleado se destinara al trabajo clínico guiado y supervisado y el $25 \%$ restante a seminarios, sesiones clínicas, proyectos de investigación, seguimiento de contenidos monográficos en las revistas internacionales especializadas, etc.? Pues que, probablemente, no estarían muchos de ellos bajo la espada de Damocles, tanto en lo que atañe a la preparación del PIR como en lo que respecta a la práctica clínica privada.

Pero se obvió lo que era esencial en este ámbito de competencia profesional y hoy son muchos los que andan azacanados y confusos y se lamentan de la ausencia de estas iniciativas. Es obvio que la formación de un psicólogo clínico forzosamente ha de ser costosa. Pero mucho más costoso es la impericia y la ineficacia profesionales. Mucho más costosa es también, por ejemplo, la matrícula de los que acceden a los estudios de postgrado de una Business School y, sin embargo, para eso sí que hay medios, de los que hoy se beneficia un numeroso grupo de licenciados en Económicas, Derecho, etc.

Hay aquí un injusto agravio comparativo, que resulta evidente entre unos y otros estudiosos. La razón de ello no está en que el Estado destine más recursos a estos últimos que a aquéllos, sino que los primeros han apelado a las empresas, es decir, a otras fuentes de recursos y firanciación. Sin embargo, pienso que en los psicólogos que se decidan por la Psicología Clínica no ha de cumplirse aquello que afirmara Juvenal de que "todos quieren saber, pero ninguno pagar".

Sea como fuere, y de atenernos a los hechos, lo que de aquí resulta es que en la sociedad actual se prioriza la formación empresarial al mismo tiempo que se escatiman los recursos necesarios para la formación de quienes han de atender y entender de los problemas de salud. ¿Es que acaso es más importante la vida 
económica que la salud personal de los ciudadanos? Por supuesto que -lejos de todo eufemismo y manipulación del lenguaje- no puede haber una sin la otra. Pero, por eso precisamente, es indispensable que haya ambas, y para ello es imprescindible presupuestar el monto necesario, a fin de que se provea de los fondos que hacen posible la formación en Psicología Clínica.

En las Universidades privadas suelen darse otros problemas de diversa naturaleza, en lo que respecta a la Psicología Clínica. En efecto, es cierto que la ratio profesor/alumno es mucho más alta que en las Universidades públicas, lo que sin duda alguna facilita en aquéllas la organización de las enseñanzas prácticas, probablemente con un mayor rigor, empeño y dedicación temporal.

Pero hay otras cuestiones en las que vale la pena reparar. Es preciso estar advertido respecto del Profesor que destina todo su celo académico a sólo la preparación de las clases y a ejercer de tutor-protector de cada uno de sus alumnos $y$, por el momento, a nada más.

Esto ha de conducir a medio plazo al enquilosamiento de la enseñanza universitaria, es decir, a transformar la Universidad en una mera academia, en la que se facilita tanto el aprendizaje a los alumnos que se acaba por sustituirlos en lo que podrían -y hasta deberían hacer- $y$, por consiguiente, se les anula como personas.

Un Profesor no debiera hacer por el alumno lo que el alumno puede hacer por sí mismo. La sustitución protectora del profesorado finaliza en la anulación personalizada del alumnado. Un Profesor, en cambio, habría de ascender a sus alumnos hasta su propia altura en los conocimientos que imparte, y establecer a esa altura el debate con ellos, aunque para ello haya que aumentar de forma significativa su nivel de exigencia.

De otra parte, si el Profesor no investiga se transforma en un "vendedor de palabras", pronunciadas en el mejor de los casos a través de una boca sublime, es decir, en un buen recitador de lo que otros han pensado, investigado y escrito.

Lo lógico y exigible es que el Profesor investigue en aquello que enseña -por eso no puede actuar de "comodín" y explicar cada curso una asignatura diferente, por la sencilla razón de que cada curso no se investiga sobre una diferente disciplina-, de manera que con 
la continuidad, el esfuerzo y la paciencia se transforme en un experto en el tema, capaz de apoyar el aprendizaje de lo que enseña a sus alumnos con sus publicaciones científicas o su propio texto $y$ monografías.

En los estudios de postgrado la cercanía de la realidad clínica constituye el mejor contexto, el contexto por antonomasia donde aprender a practicar el comportamiento de respeto a la dignidad de las personas, al mismo tiempo que la prudencia con que ha de ser tratada cualquier cuestión científica. Esta cuestión es hoy fundamental si no se desea mantener una actitud servil o temerosa respecto de la ciencia. "La opinión pública se ha dividido en dos sectores: los que piensan que la ciencia puede hacer cualquier cosa, y aquellos que temen que lo haga". Estas palabras de quien fuera Secretario de Estado de EE. UU., Dixy Lee Ray (1973), constituyen, lamentablemente, otra de las profecías cumplidas.

\section{LAS TIERRAS IGNOTAS DE LA PSICOLOGÍA CLIÍNICA}

Lo que hoy entendemos por Psicología Clínica es, en mi opinión, apenas una muy modesta parcela de la indefinida y casi ilimitada superficie de esta extensa actividad. Es cierto que su actual significado se ha restringido a lo que podríamos denominar "núcleo duro" de esta especialidad. Es decir, se ha magnificado todo lo relativo a los trastornos psíquicos $y$, por ahora, se ha condenado al olvido casi todo lo demás.

El reduccionismo que comporta tan desacertada opción ha prolongado y ahondado las discrepancias entre el psicólogo y ciertos profesionales, sin que ninguno de ellos gane algo y todos pierdan. De estos supuestos enfrentamientos -en ocasiones ficticios y otras veces mejor fundamentados $y$ atenidos a la realidad- el autor dispone de cierta experiencia, en la que aquí no es pertinente entrar y cuya exposición se reserva para otra ocasión y otro contexto.

En todo caso, es necesario abrir los horizontes de la Psicología Clínica. Hay muchas "tierras ignotas" en ella que el psicólogo clínico todavía no se ha atrevido ni siquiera a explorar. Si como se ha observado líneas atrás, lo propio del psicólogo clínico es ir al en- 
cuentro de cada persona doliente, allí donde se demuestre que los factores psicológicos son la causa, sostén, condicionamiento, mantenimiento o perpetuación de su malestar personal, no se entiende como ha podido marginar sus conocimientos y excluir de su labor asistencial a tantas personas que sufren.

El 11-M, lamentablemente, constituyó un hito sociológico -acaso el más relevante- en la historia de la Psicología Clínica española y de la aceptación social de quienes se dedican a ella. En esa fecha se confunde la tragedia y la clínica, la eficaz responsabilidad de los psicólogos y el inicio de su reconocimiento social. En opinión de quien esto escribe hay muchos "11-M" ignotos, que es preciso desvelar y sacarlos a la superficie del quehacer clínico, para allí atenderlos y tratarlos como se merecen.

Aunque sólo sea con ánimo de ayudar a reflexionar a los lectores de esta colaboración, se formularán a continuación algunas cuestiones que, es de desear, ayuden a las próximas generaciones de psicólogos clínicos a encaminarse hacia esas "tierras" todavía desconocidas de la Psicología Clínica. Son -preciso es reconocerloinquietudes del autor que ha procurado compartir con otros investigadores, sin que la travesía de su vida -la vida no da para tantole haya permitido entrar como debiera en su dilucidación.

¿Cuántos psicólogos clínicos trabajan hoy con enfermos crónicos, con independencia de cuál sea la enfermedad que sufren?, ¿es que acaso no podrían mejorar su calidad de vida, su voluntad de recuperación, su adaptación a las limitaciones y secuelas que en ellos ha dejado la enfermedad?, ¿a cuánto asciende el número de pacientes afectados por estas enfermedades, con independencia de cuál sea su edad?, ¿es que tal vez está demostrada la inexistencia de factores psicológicos en lo que se refiere a la hipertensión, las cefaleas, la úlcera gástrica, la diabetes, los trastornos vasculares, los accidentes politraumáticos, las hemiplejías, los enfermos terminales, la recuperación del coma y las alteraciones de los estados de conciencia, los pacientes oncológicos, el asma, los pacientes sometidos a hemodiálisis, los pacientes geriátricos, etc.?, ¿es que no hay quizás bibliografía científica disponible acerca de ello?, ¿para cuándo se dejará el diseño de los necesarios programas al respecto? 
¿Puede el psicólogo clínico hacer algo más por el paciente y su entorno familiar?, ¿qué es lo que suele suceder con los hijos de padres depresivos?, ¿y con los padres de niños oncológicos, con autismo, espina bífida, retraso mental, etc.?, ¿puede ayudarse a estos padres a fin de que su salud no se quebrante, conlleven mejor las frustraciones e incidan de forma más terapéutica en el comportamiento de su hijos enfermos?, ¿pueden diseñarse programas de preparación para el ingreso hospitalario y las intervenciones quirúrgicas, de forma que se prevenga la ansiedad y depresión de los afectados, se minimicen sus efectos nocivos y disminuya el tiempo de hospitalización?, ¿en cuánto se reduciría el gasto con ello?, ¿sería suficiente ese ahorro para que el hospital contratara al psicólogo clínico, a fin de generalizar el programa, una vez se haya demostrado su eficacia?

La propuesta que aquí se hace no consiste tanto en solicitar que se dote de un psicólogo a cada uno de los Servicios clínicos, cualquiera que fueren las competencias y el contenido de su especialidad -cosa probablemente utópica en el actual Servicio Nacional de Salud-, como la de motivar al Psicólogo Clínico a que se forme e investigue en los numerosos contextos hospitalarios y ambulatorios en que puede probarse la eficacia -económica, asistencial y, sobre todo, humana- de su trabajo.

¿Cuántos psicólogos clínicos trabajan hoy en estudios epidemiológicos acerca de las muy variadas alteraciones psíquicas?, ¿se pueden prevenir estas alteraciones cuando se ignora incluso hasta cuáles son su prevalencia e incidencia?, ¿se han llevado a cabo, en este contexto, algunos programas preventivos que hayan demostrado ser eficaces?

At last but not least, es mucho lo que resta por hacer en el especifico y necesario ámbito de las psicoterapias e intervenciones del psicólogo clínico. La formación de psicoterapeutas y la evaluación de la eficacia diferencial de las diversas terapias, así como de en qué situaciones, trastornos y personas están más indicada cada una de ellas, constituyen contenidos relevantes que piden ser atendidos en los foros y debates científicos que para estos fines se organicen, siempre que lo que alli se formule vaya precedido $y / o$ acompañado de la necesaria comprobación empírica. 
Todavia son muchos los que sostienen que no hay psicoterapias sino psicoterapeutas; que el psicoterapeuta nace y no se hace; que la eficacia de una intervención está condicionada más por la personalidad del psicólogo que la realiza que por el tipo de estrategia terapéutica empleada, etc. Como puede observarse, no deja de haber un halo misterioso en todo esto, lo que por otra parte es comprensible, dadas las dificultades que entrañan tales evaluaciones. Pero es ésta una cuestión esencial que ha de esclarecerse, porque atañe tanto a la credibilidad social del psicólogo clínico como al objetivo progreso científico de la actividad clínica a la que se dedica.

Sería conveniente en este punto realizar ciertas indagaciones que no resultan en la actualidad prescindibles y que exigirian ser atendidas. Me refiero, claro está, al perfil psicológico que sería más adecuado para quienes aspiran a dedicarse al ejercicio profesional de la Psicología Clínica; a los factores de los que depende el grado de motivación y satisfacción de estos profesionales; a la evaluación de los diversos programas existentes para la formación de clínicos y terapeutas; a los mínimos imprescindibles que deben satisfacerse antes de habilitar a un psicólogo para el ejercicio de la práctica clínica; a los requisitos de los programas institucionales de mantenimiento en la formación y actualización de conocimientos de estos profesionales; a los estudios comparativos, multicéntricos e interuniversitarios realizados, en lo relativo a los resultados obtenidos por estos profesionales; etc.

Como puede comprobarse, es mucho lo que todavía queda por hacer en el ámbito de la Psicología Clínica española. Es lógico que sea así, sobre todo si nos atenemos al escaso tiempo transcurrido desde que se comenzó a impartir estas enseñanzas en nuestro país, tiempo que con una medida más humana me atrevería a establecer en menos de dos generaciones de Profesores.

Pero eso no ha de justificar el retraso en la implantación de esta profesión en nuestra sociedad, sino que ha de avivar la motivación y el paso de quienes a ella nos dedicamos. Pues como reza uno de los aforismos hipocráticos, "la vida es breve, la ciencia larga, la ocasión huidiza [también la actual], el experimento peligroso, el juicio difícil". 


\section{BIBLIOGRAFÍA}

Bacon, F. (1984). Novum Organum, libro primero, aforismo 129, Orbis, Barcelona, p. 81.

Faraday, M. (sin fecha). Citado en Experimental Researches in Electricity, Dover, Nueva York.

Gluckman, M. (1965). Politics, Law and Ritual, Mentor, Nueva York, 1965, 60.

Gould, S. J. (1984). Conferencia sobre evolución. Cambridge, 1984.

Hipócrates. Aforismos 1.

Juvenal, $7,157$.

Ollero Tassara, A. (1985). Qué hacemos con la Universidad. Instituto de Estudios Económicos. Madrid, p.77.

Ray, D. L. (1973). New Scientist, 59, 853, p. 14.

Varrón. Sententiae, 56.

Varrón. De Re Rustica, 2, 1, 2. 